\title{
Cell division cycle associated 5 promotes colorectal cancer progression by activating the ERK signaling pathway
}

Aling Shen 1,2,3, Liya Liu ${ }^{3}$, Hongwei Chen ${ }^{1,2}$, Fei Qi ${ }^{1,2}$, Yue Huang ${ }^{1,2}$, Jiumao Lin ${ }^{1,2}$, Thomas Joseph Sferra ${ }^{3}$, Senthilkumar Sankararaman ${ }^{3}$, Lihui Wei ${ }^{1,2}$, Jianfeng Chu ${ }^{1,2}$, Youqin Chen ${ }^{1,2,3}$ and Jun Peng ${ }^{1,2}$

\begin{abstract}
Cell division cycle associated 5 (CDCA5) is implicated in the development and progression of a variety of human cancers. Functional significance of CDCA5 in colorectal cancer (CRC), however, has not been investigated. Using a combination of on-line data mining, biochemistry, and molecular biology, we examined the potential oncogenic activity of CDCA5 and the underlying mechanisms. Experiments with human tissue sample showed increased CDCA5 expression in CRC vs. in noncancerous adjacent tissue, and association of CDCA5 upregulation in CRC tissues with shorter patient survival. Also, representative CRC cell-lines had higher CDCA5 expression vs. fetal colonic mucosal cells. CDCA5 knockdown using lentivirus-mediated shRNA inhibited the proliferation and induced apoptosis in cultured HCT116 and HT-29 cells, and suppressed the growth of xenograft in nude mice. CDCA5 knockdown decreased the expression of CDK1 and CyclinB1, increased caspase-3 activity, cleaved PARP and the Bax/Bcl-2 ratio. CDCA5 knockdown also significantly decreased phosphorylation of ERK $1 / 2$ and expression of c-jun. Taken together, these findings suggest a significant role in CRC progression of CRC, likely by activating the ERK signaling pathway.
\end{abstract}

\section{Introduction}

Colorectal cancer (CRC) is the third leading cause of cancer-related death worldwide ${ }^{1}$. Despite recent advances in early diagnosis of and treatments for CRC, patient mortality remains high.

Uncontrolled growth is a key feature of cancers ${ }^{2,3}$. Accordingly, suppressing the proliferation of cancer cells represent an important strategy in anticancer treatment. In eukaryotic cells, proliferation is primarily regulated by cell cycle ${ }^{4}$ that contains three major checkpoints-one at the G1-S transition and two at G2-M transition ${ }^{5}$. Sister

\footnotetext{
Correspondence: Youqin Chen (yxc571@case.edu) or Jun Peng (pjunlab@hotmail.com)

${ }^{1}$ Academy of Integrative Medicine, Fujian University of Traditional Chinese Medicine, 1 Qiuyang Road, Minhou Shangjie, Fuzhou, Fujian 350122, China

${ }^{2}$ Fujian Key Laboratory of Integrative Medicine on Geriatrics, Fujian University of Traditional Chinese Medicine, 1 Qiuyang Road, Minhou Shangjie, Fuzhou, Fujian 350122, China

Full list of author information is available at the end of the article.

These author contributed equally: A. Shen, L. Liu
}

chromatid cohesion in the $\mathrm{S}$ phase and segregation of sister chromatids in the anaphase of mitosis are two important processes during cell mitosis that safeguard the accurate separation of parental chromosomes into two daughter cells. Human CDCA5 (cell division cycle associated 5), also known as sororin, was originally identified as a substrate of the anaphase-promoting complex ${ }^{6-8}$. CDCA5 is required for stable binding of cohesin to chromatid in the $\mathrm{S}$ and $\mathrm{G} 2 / \mathrm{M}$ phases and is degraded through anaphase-promoting complex-dependent ubiquitination in the G0/G1 phase ${ }^{6-9}$.

CDCA5 has been found to be overexpressed, and correlated with poor prognosis in several human cancers, including lung carcinomas, urothelial carcinoma, and oral squamous cell carcinoma ${ }^{10-14}$. Consistent with CDCA5 overexpression in cancer cells, knockdown of CDCA5 could inhibit cancer growth by arresting the cell cycle in the G2/M phase and promoting apoptosis ${ }^{11,14}$.

\section{(c) The Author(s) 2019}

(c) (i) Open Access This article is licensed under a Creative Commons Attribution 4.0 International License, which permits use, sharing, adaptation, distribution and reproduction c. in any medium or format, as long as you give appropriate credit to the original author(s) and the source, provide a link to the Creative Commons license, and indicate if changes were made. The images or other third party material in this article are included in the article's Creative Commons license, unless indicated otherwise in a credit line to the material. If material is not included in the article's Creative Commons license and your intended use is not permitted by statutory regulation or exceeds the permitted use, you will need to obtain permission directly from the copyright holder. To view a copy of this license, visit http://creativecommons.org/licenses/by/4.0/. 
In the current study, we examined whether CDCA5 is also implicated in the development and progression of CRC. First, we compared gene-expression profile in primary CRC lesions vs. matched healthy tissues. Analysis of the differentially expressed genes using RNA interference and high-content screening identified CDCA5 as a potential target. We then conducted a series of experiments using representative CRC cell lines as well as xenograft nude mice models to examine the functional role of CDCA5.

\section{Results}

\section{CDCA5 is highly expressed in CRC tissues and cultured cells}

Quantitative real-time polymerase chain reaction (qPCR) assay in 50 pairs of primary CRC lesions and adjacent noncancerous tissues revealed higher CDCA5 mRNA level in CRC tissue (Fig. 1a). Such result was verified by immunohistochemical (IHC)-based tissue microarray (TMA) of 73 pairs of primary CRC lesions and adjacent noncancerous tissue (Fig. 1b). Similar results were obtained with online data mining using the R2 Bioinformatic Platform (http://r2.amc.nl) and TCGA (https://cancergenome.nih.gov/) (Fig. 1c, d). qPCR and Western-blot analyses of cultured human CRC cell lines (Caco-2, HT-29, RKO, HCT116, and HCT-8) also showed significantly higher CDCA5 expression in CRC cells than in fetal colonic mucosal cells (FHC) (Fig. 1e, f; $P<0.05$ ).

\section{CDCA5 overexpression is associated with poor prognosis in CRC patients}

Analysis of 92 CRC patients showed higher overall survival rate in subjects with higher CDCA5 expression (as determined with IHC-based TMA) (Fig. 2a, b; $P<$ $0.05)$. High-CDCA5 expression was also associated with more advanced $\mathrm{N}$ stage (Supplementary Table 1; $P<0.05$ ). Online data mining using the R2 Bioinformatic Platform (http://r2.amc.nl) also revealed an association between high-CDCA5 expression and poor overall survival in CRC patients (Fig. 2c; $P<0.05$ ).

\section{CDCA5 knockdown inhibits the proliferation of cultured CRC cells}

We next constructed three lentiviral shRNAs specific for CDCA5. qPCR and Western-blot analyses revealed significantly reduced CDCA 5 mRNA (Supplementary Fig. 1A, B) and protein (Supplementary Fig. 1C) levels with all three shRNAs in both HCT116 cells and HT-29 cells (constitutively expressing CDCA5; Fig. 1e, f). The shCDCA5-2 construct was used for subsequent experiments, and referred to as "sh-CDCA5".

CDCA5 knockdown in cultured HCT116 and HT-29 cells significantly reduced cell number (Fig. 3b; $P<0.05$ vs. sh-Ctrl), cell viability (Fig. 3 c; $P<0.05$ ) and colony formation (Fig. 3d; $P<0.05$ ). Cell cycle analysis showed decreased cell percentage in the G0/G1 phase and increased percentage in the G2/M phase in both HCT116 and HT-29 cells (Fig. 4a; $P<0.05$ vs. sh-Ctrl). The expression of G2/M-related proteins CDK1 and CyclinB1 was decreased by CDCA5 knockdown in HCT116 cells (Fig. 4b).

\section{CDCA5 knockdown induces apoptosis in cultured CRC cells}

Hoechst staining revealed typical apoptotic morphological features (e.g., condensed chromatin and fragmented nuclei) in HCT116 cells upon CDCA5 knockdown (Fig. 5a). Flow cytometry with Annexin V staining increased the percentage of apoptotic cells (Fig. $5 \mathrm{~b} ; P<0.05$ vs. shCtrl). Caspase- 3 activity was increased (Fig. 5 c; $P<0.05$ vs. sh-Ctrl). Western-blot analysis showed increased Bax/Bcl2 ratio and increased expression of cleaved Poly ADPribose polymerase (PARP) (Fig. $5 \mathrm{~d} ; P<0.05$ vs. sh-Ctrl).

\section{CDCA5 suppresses the growth of CRC tumor xenograft}

Upon subcutaneous implantation, HCT116 xenograft transduced with sh-CDCA5 grew at a much lower rate vs. the control (Fig. 6a for tumor volume; Fig. 6b for intratumoral green fluorescent protein (GFP) fluorescence and Fig. 6c for tumor weight; $P<0.05$ for all). IHC showed that CDCA5 knockdown (Fig. 7a; $P<0.05$ vs. sh-Ctrl) significantly decreased proliferating cell nuclear antigen (PCNA) expression in xenograft tumor (Fig. 7b; $P<0.05$ vs. sh-Ctrl). Terminal deoxynucleotidyl transferase dUTP nick end labeling (TUNEL) assay showed increased cell apoptosis in HCT116 xenograft upon CDCA5 knockdown (Fig. 7c; $P<0.05$ vs. sh-Ctrl).

\section{CDCA5 knockdown suppresses the activation of ERK signaling pathway}

Activation of the ERK pathway plays a pivotal role in the pathogenesis of $\mathrm{CRC}^{15-18}$. In our experiment, CDCA5 knockdown significantly decreased the phosphorylation of ERK1/2 and c-jun expression in cultured HCT116 cells (Fig. 8; $P<0.05$ ).

\section{Discussion}

The first major finding in the current study is the overexpression of CDCA5 in CRC tissues vs. in adjacent noncancerous tissues. The overexpression is apparent in our own study cohort and supported by the online datasets in the R2 application website and TCGA. Overexpression of CDCA5 was also found in several representative CRC cell lines vs. in normal human FHC, suggesting that CDCA5 overexpression is a common event in CRC. Survival analysis based on TMA of CRC samples and online public database associated higher CDCA5 expression with poorer patient prognosis. These findings are largely consistent with the studies in lung 


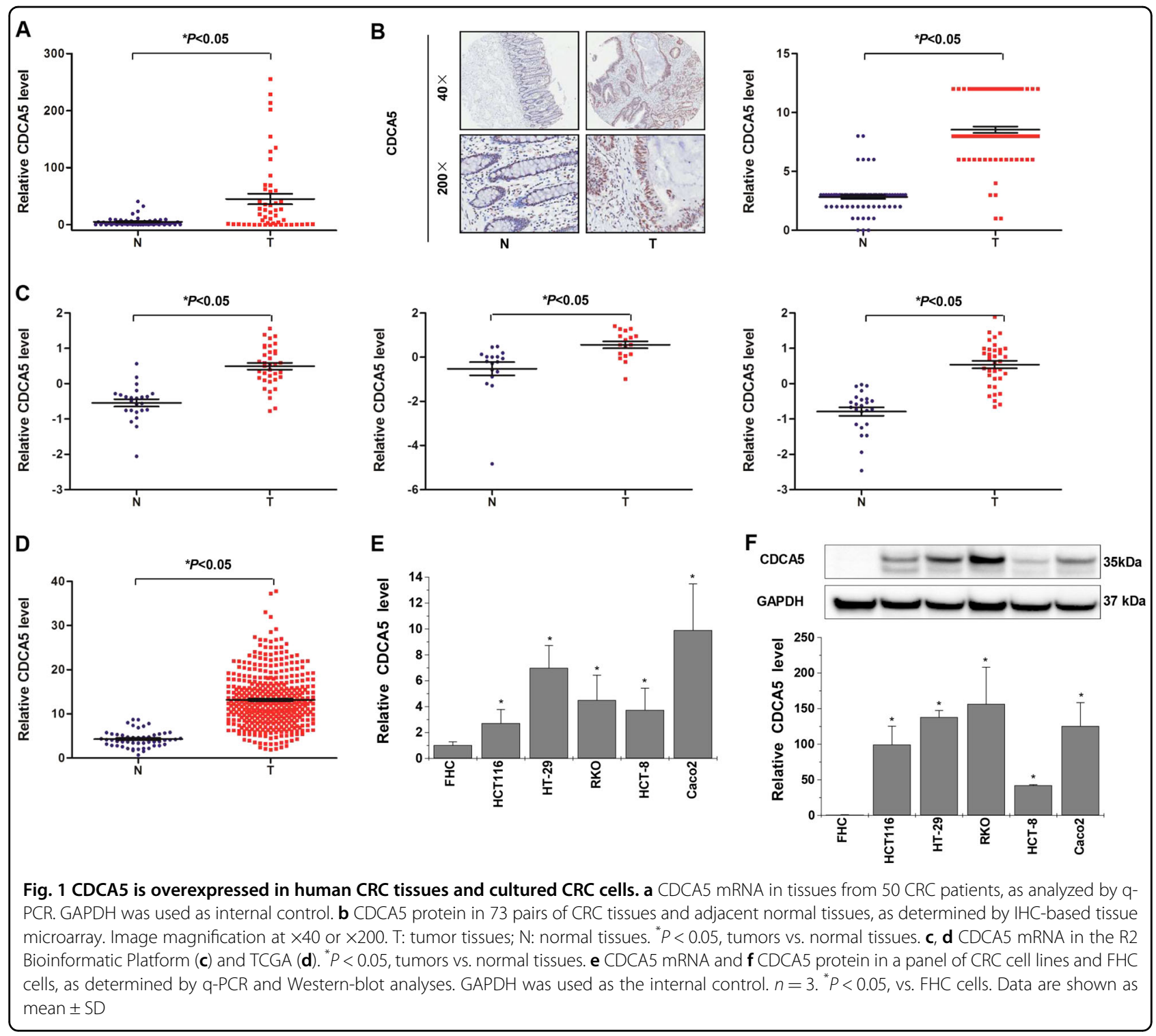

carcinoma, urinary bladder carcinoma, and oral squamous cell carcinoma ${ }^{11,13,14}$, and suggested a functional role for CDCA5 for a broader range of cancers. The results must be interpreted with caution due to the relatively small sample size and the inclusion of only Chinese patients. The prognostic significance of CDCA5 requires further validation by studies of larger sample size with diverse ethnic background.

Previous studies indicated that CDCA5 knockdown could inhibit the growth of lung carcinoma and oral squamous cell carcinoma cell ${ }^{11,14}$. In the current study, we knocked down the expression of CDCA5 in two representative CRC cell lines (HCT116 and HT-29) using a lentivirus-mediated shRNA, and found decreased cell growth in vitro as evidenced by decreased cell number, viability, and survival rate. Using a nude mouse xenograft model bearing HCT116 or HT-29 cells transduced with sh-CDCA5 lentivirus, we found that CDCA5 knockdown could markedly decrease tumor growth. Taken together, these findings suggest an oncogenic activity of endogenous CDCA5 in CRC.

Proliferation of eukaryotic cell is primarily mediated by cell cycle. G2/M progression, one of the main checkpoints, is closely modulated by the protein complex of CyclinB1/CDK $1{ }^{19,20}$. CDCA5 is required for stable binding of cohesion to chromatid in the $\mathrm{G} 2 / \mathrm{M}$ phase and plays an essential role in cell cycle regulation ${ }^{6-9}$. Consistent with a previous study in oral squamous cell carcinoma ${ }^{14}$, we found blocked G2/M progression upon CDCA5 knockdown in both HCT116 and HT-29 cells. CDCA5 knockdown also significantly decreased CyclinB1 and CDK1 expression. Mitochondrion-dependent apoptosis is 

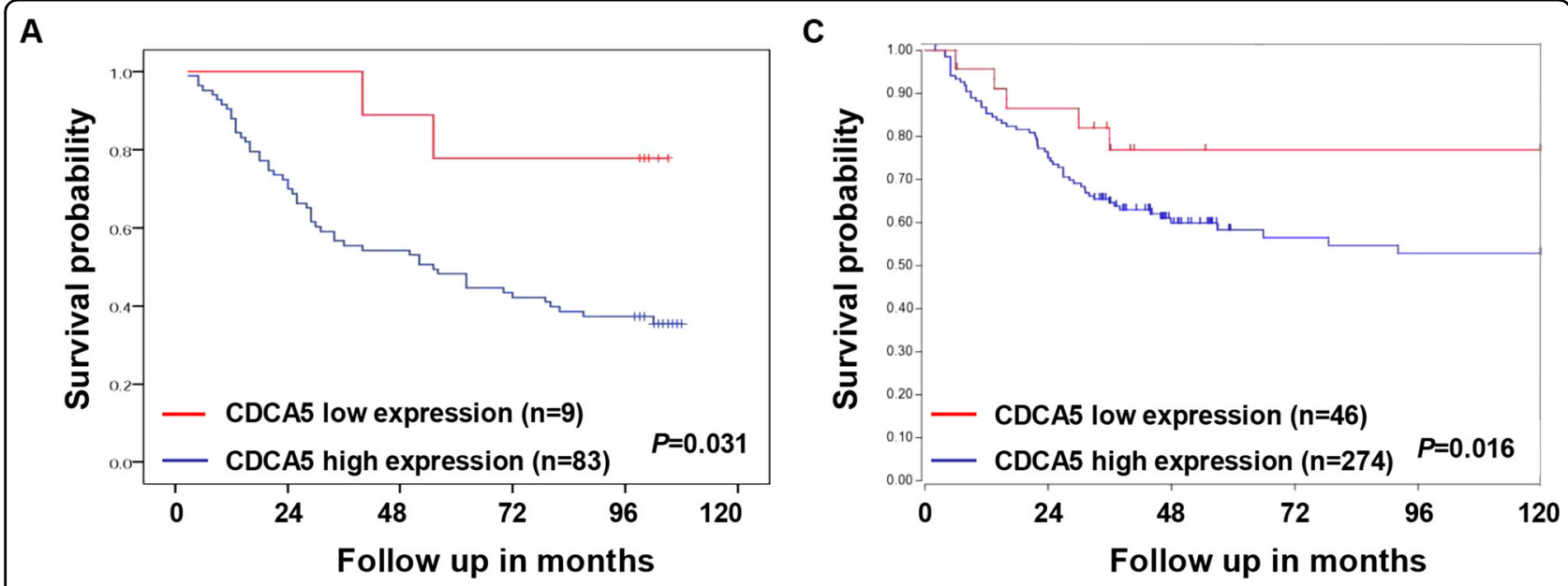

B CDCA5 low expression

CDCA5 high expression
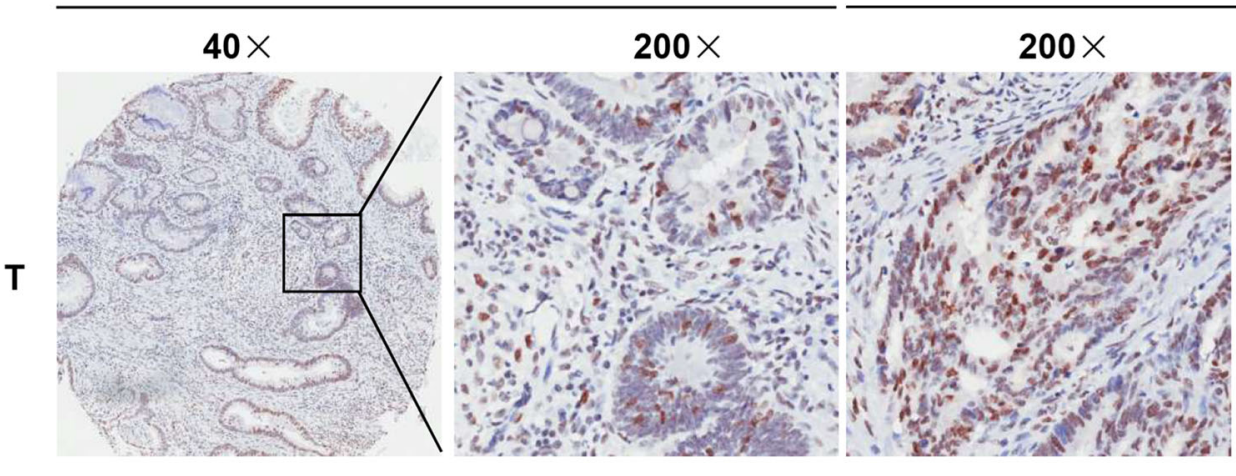

$40 \times$
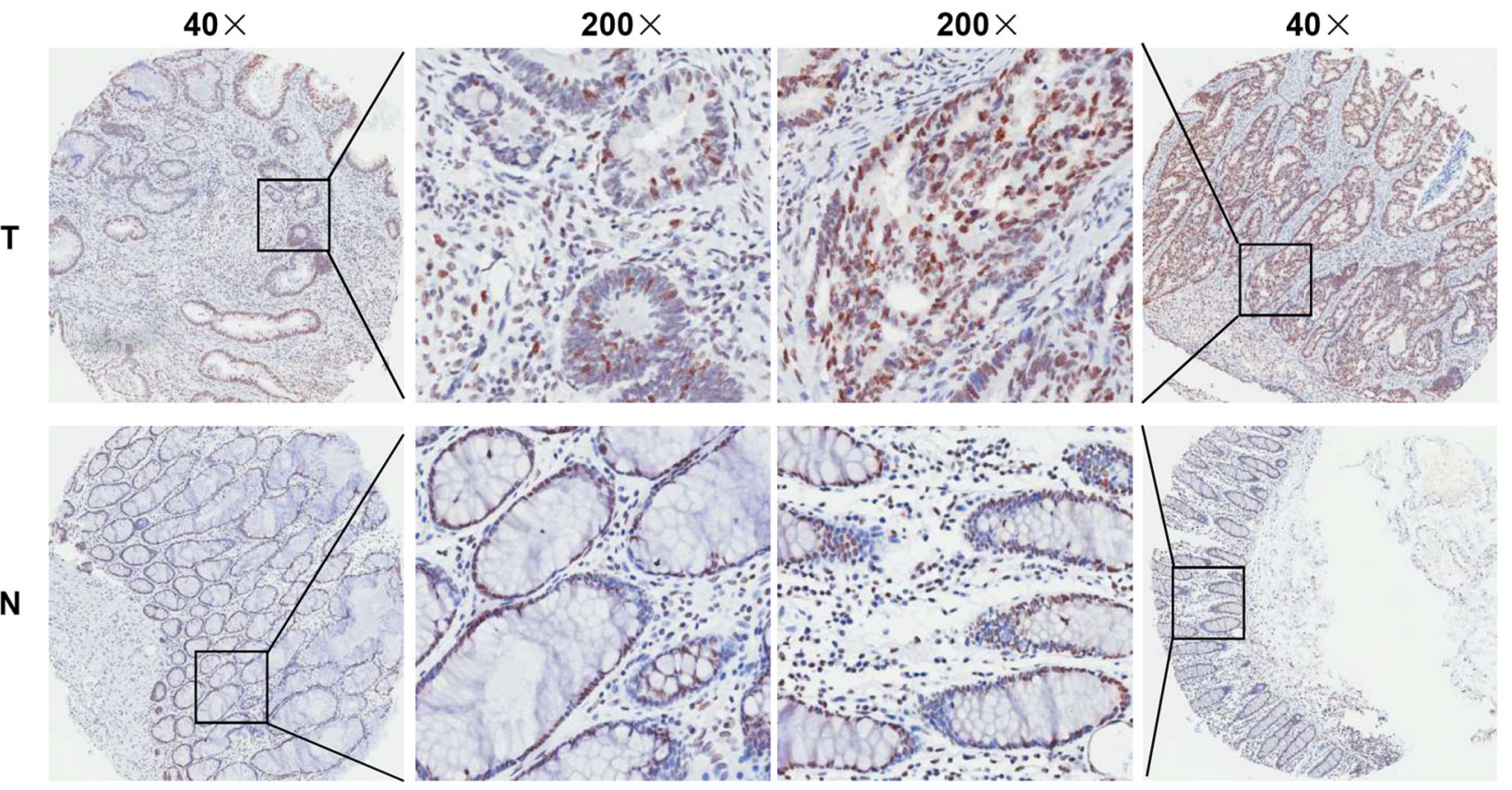

Fig. 2 Association between CDCA5 overexpression and poor patient prognosis. a Correlation between CDCA5 protein (IHC-based TMA) and patient survival, was analyzed with Kaplan-Meier plots in 92 CRC patients $(P<0.05)$. b Representative images of high- or low-CDCA5 expression. Magnification at $\times 40$ or $\times 200$. c Survival analysis based on CDCA5 expression in 320 CRC patients (GEO ID: GSE24551) from a public clinical microarray dataset $\mathrm{R} 2$ bioinformatic platform $(P<0.05)$. Survival was analyzed with log-rank test

a major apoptotic pathway. PARP, a protein essential for DNA repair, could be cleaved by caspases during the process of apoptosis. Accordingly, cleaved PARP is commonly used as an indicator of apoptosis. Bcl-2 family members, including antiapoptotic Bcl-2 and proapoptotic $\mathrm{Bax}^{21,22}$, are critical mediators of mitochondriondependent apoptosis. Aberrant expression of $\mathrm{Bcl}-2$ family proteins is commonly found in cancers. In this study, we found that CDCA5 knockdown-induced apoptosis in HCT116 cells. Colorimetric assay and western-blot analysis showed increased caspase-3 activity, PARP expression and $\mathrm{Bax} / \mathrm{Bcl}-2$ ratio in HCT116 cells in response to CDCA5 knockdown. IHC analysis and TUNEL assay in xenograft models confirmed the suppression of cell proliferation and promotion of cell apoptosis by CDCA5 knockdown. These findings indicate that CDCA5 produces oncogenic activity by disrupting the balance of proliferation/apoptosis in cancer cells. 


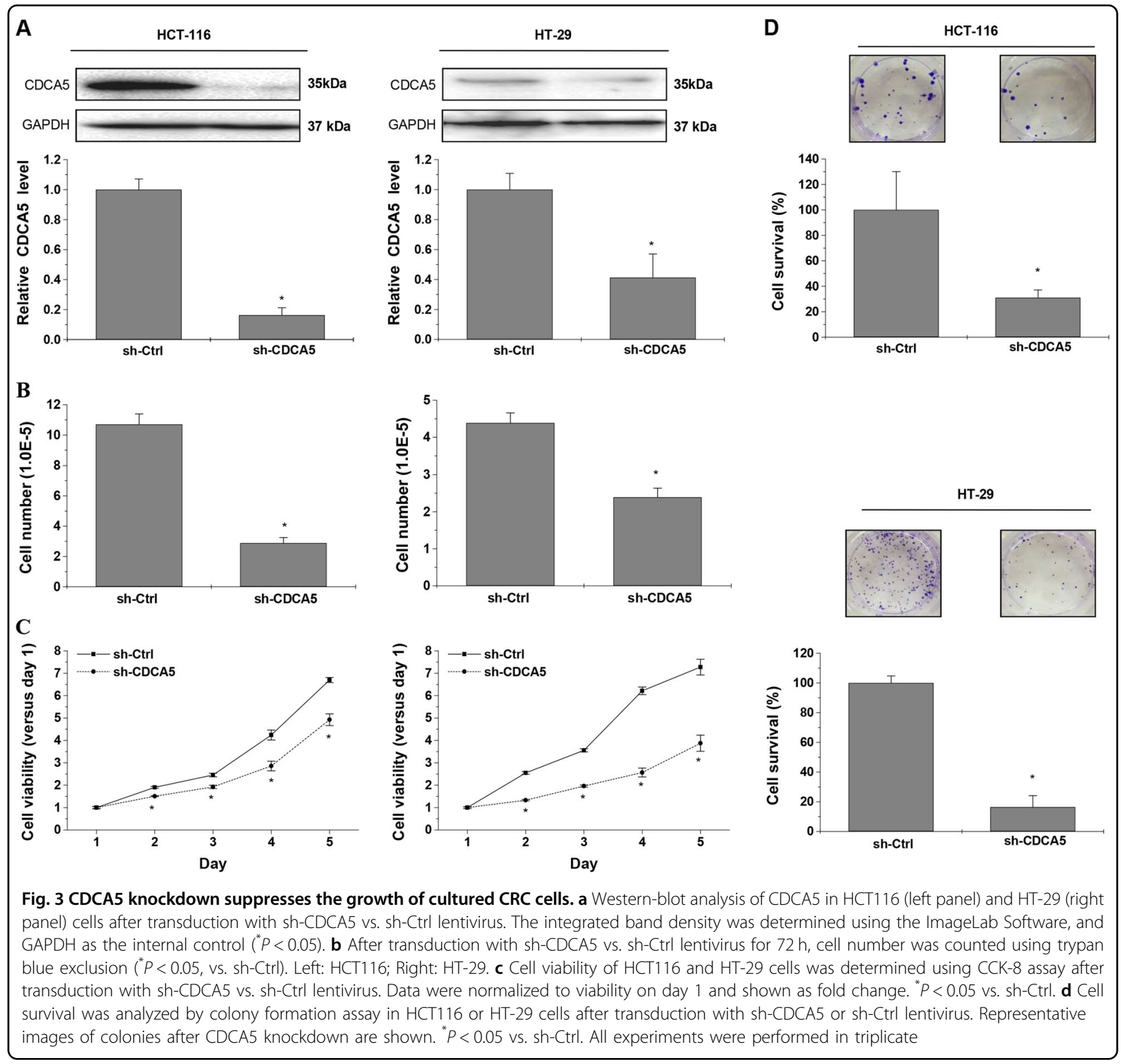

However, underlying mechanism of CDCA5 knockdown on suppression tumor growth of CRC cells need to be explored in future studies.

The ERK signaling pathway plays an important role in a variety of cellular processes, including cell survival, cell differentiation, apoptosis, invasion, and inflammation $^{18,19,23,24}$. Activation of the ERK pathway is one of the key mechanisms for the initiation and progression in many human cancers ${ }^{15-18}$, including $\mathrm{CRC}^{25-28}$. Our finding that CDCA5 knockdown decreased the phosphorylation level of ERK1/2 and expression of c-jun in CRC cells suggests that activation of the ERK pathway contributes to the oncogenic activities of CDCA5 in CRC. However, potential effects of CDCA5 on activation of
p38MAPK, JNK, and other pathways need to be further addressed.

In summary, we found CDCA5 overexpression in CRC and an association of CDCA5 overexpression with poor patient survival. Silencing CDCA5 expression suppressed the tumor growth in vitro and in vivo, possibly by inhibiting the ERK signaling. These studies highlighted the biological function of CDCA5, and suggested that CDCA5 could be used as a potential biomarker in CRC.

\section{Methods}

\section{Patients and specimens}

A total of 50 pairs of primary CRC lesions and matched noncancerous tissues $(5 \mathrm{~cm}$ away from the margin of 


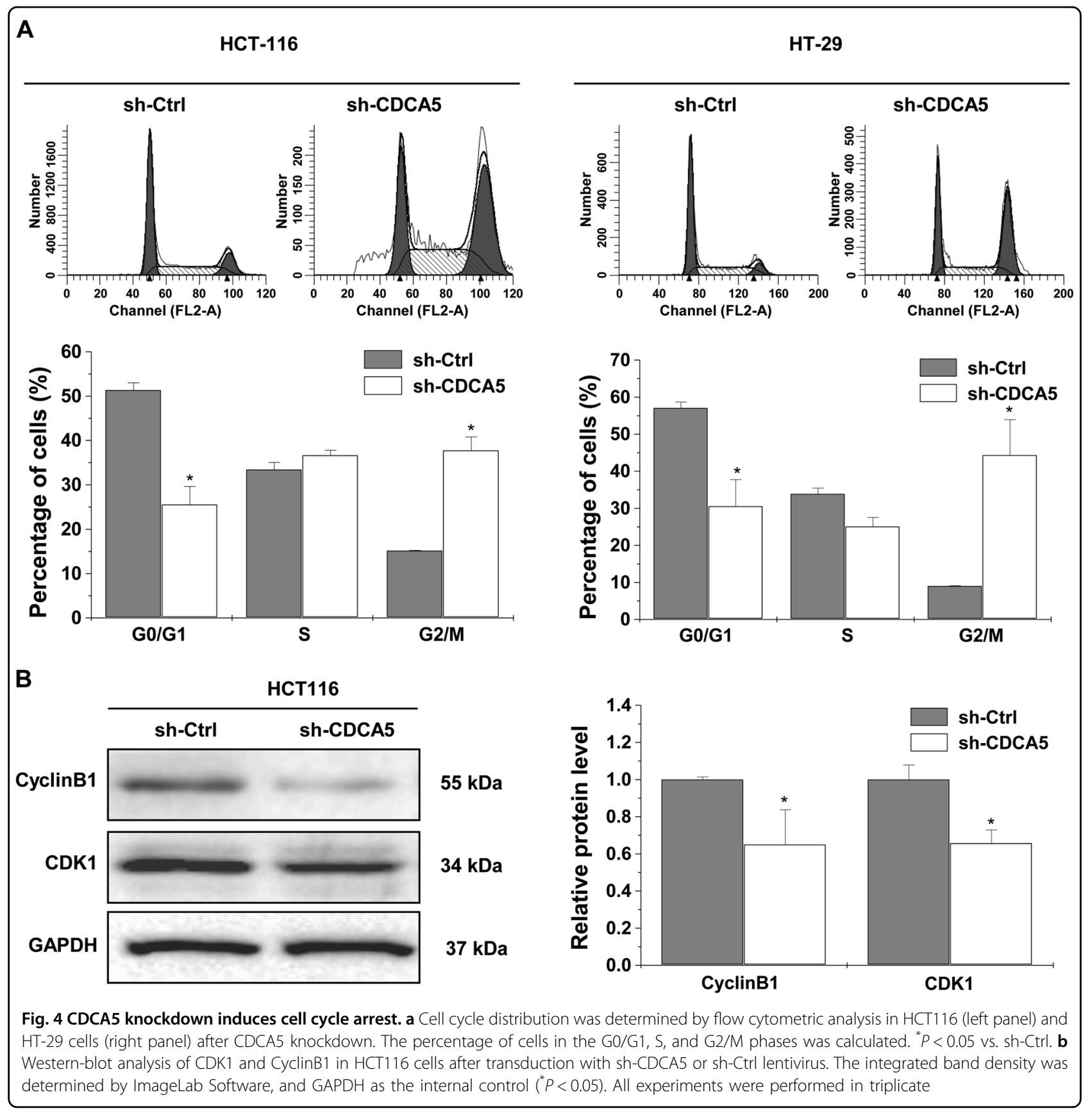

cancerous tissues) were collected from CRC patients, who received resection at First Hospital Affiliated to Fujian University of Traditional Chinese Medicine (FJTCM) or Fujian Provincial Hospital in 2013 or 2014. None of the patients received chemotherapy or radiotherapy prior to the surgery. Tissue specimens were either fixed in $4 \%$ paraformaldehyde for $24 \mathrm{~h}$ followed by paraffin embedding, or snap frozen and kept in liquid nitrogen until further use. Clinicopathologic characteristics of the patients are shown in Supplementary Table 2. The study was approved by the Ethics Committee of FJTCM and Fujian Provincial Hospital. Written informed consent was obtained from all human participants.

\section{q-PCR analysis}

Total RNA was extracted using RNAiso Plus reagent (Takara; Dalian, Liaoning, China), and reverse-transcribed $(1 \mu \mathrm{g})$ into cDNA using PrimeScript RT kit (Takara). Gene expression was examined using q-PCR with ABI 7500 Fast Real-Time PCR System (Applied Biosystems; 


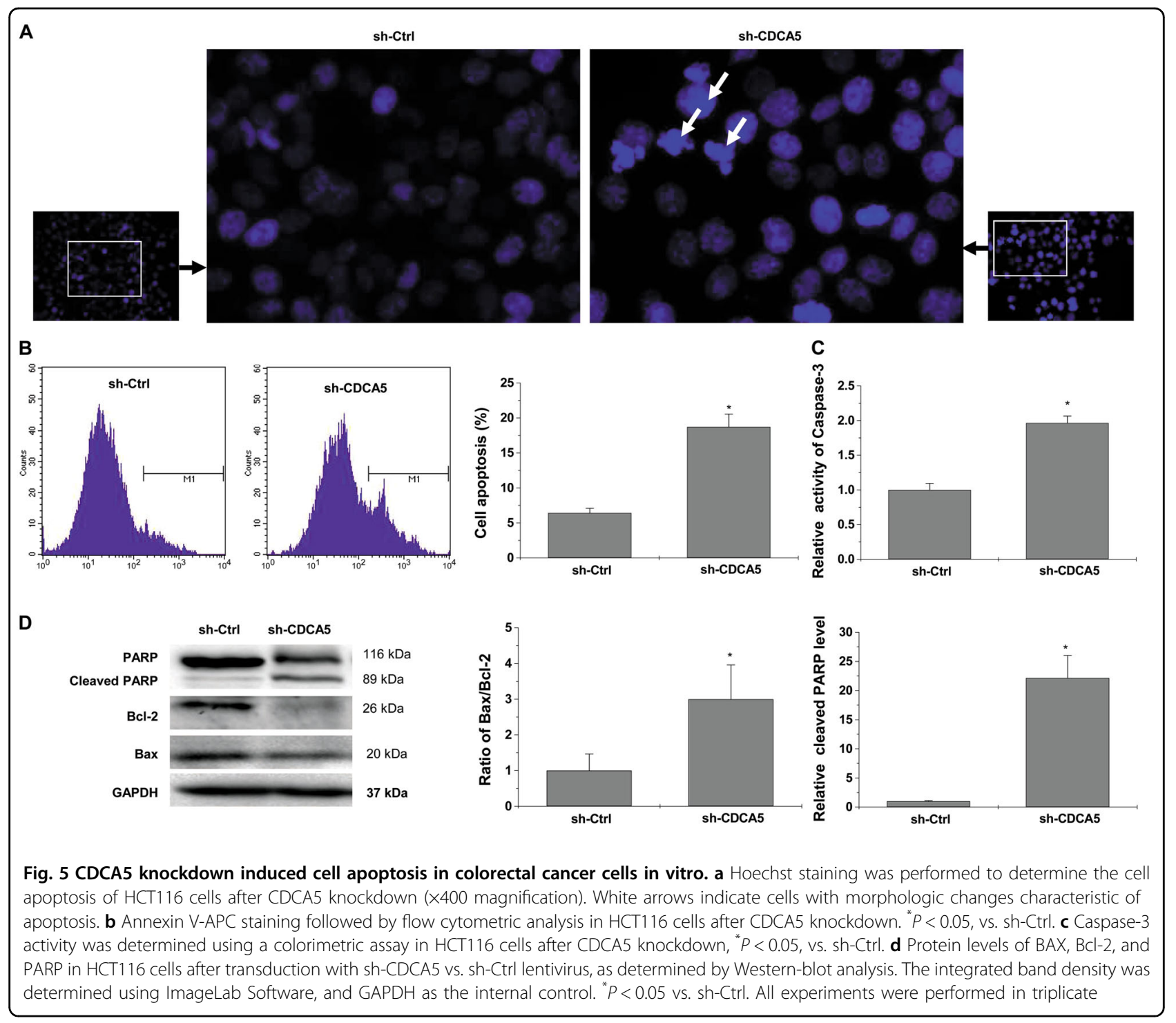

Carlsbad, CA, USA), as described previously ${ }^{29}$. Sequence of the primers are shown in Supplementary Table 3. mRNA was quantified using $2^{-\Delta \Delta C T 29}$.

\section{TMA and survival analysis}

TMA chips of CRC and control tissues were obtained from Shanghai Outdo Biotech Company (Shanghai, China; Cat\#: HColA180Su09). CDCA5 IHC was conducted using an antibody against CDCA5 (Rabbit monoclonal to CDCA5-C-terminal; dilution 1:800; Abcam, ab192237; USA), as described previously ${ }^{30}$. Scoring was carried out by two experienced pathologists blinded to tissue identity using a grading system based on staining intensity (no staining, 0; weak, 1; moderate, 2; strong, 3) and percentage of positive-staining cells (1-25\% positive, $1 ; 26-50 \%, 2$;
$51-75 \%$, 3; 76-100\%, 4) ${ }^{31}$. The final score was calculated as intensity score $\times$ percentage score. For survival analysis, CDCA5 expression in CRC tissues was classified into low (final score: 0-6) vs. high (final score: 7-12). Kaplan-Meier survival curves were plotted for high- and low-expression groups and analyzed using log-rank test.

\section{Bioinformatics analysis}

CDCA5 mRNA in CRC vs. control tissues was analyzed using the R2 Bioinformatic Platform (http://r2.amc.nl) ${ }^{32-34}$ and TCGA (https://cancergenome.nih.gov/). Kaplan-Meier analysis was performed to analyze the correlation between CDCA5 mRNA and patient survival in a dataset of 320 subjects (GEO ID: GSE24551) through R2 web application (http://r2.amc.nl) ${ }^{35}$. 


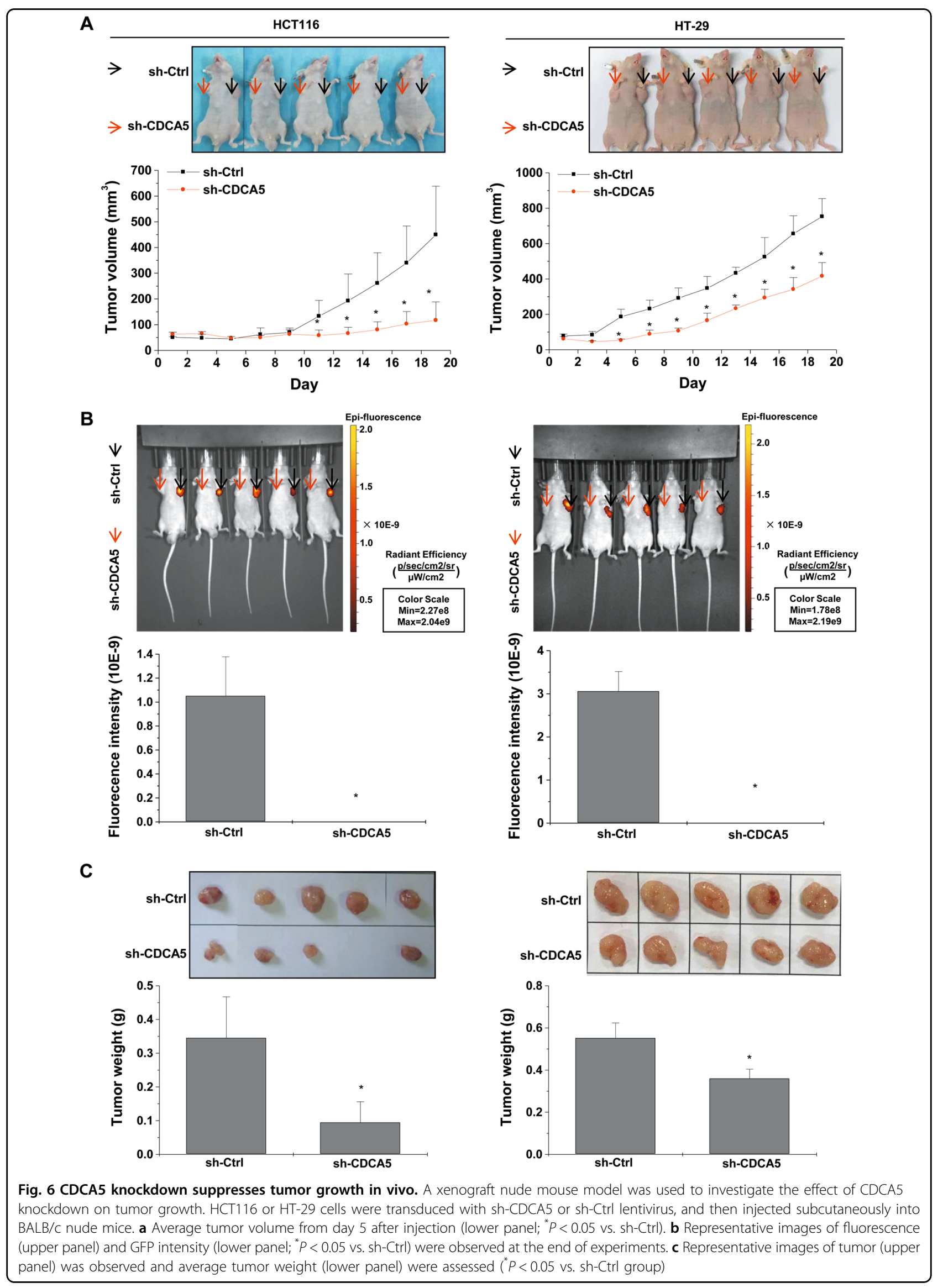



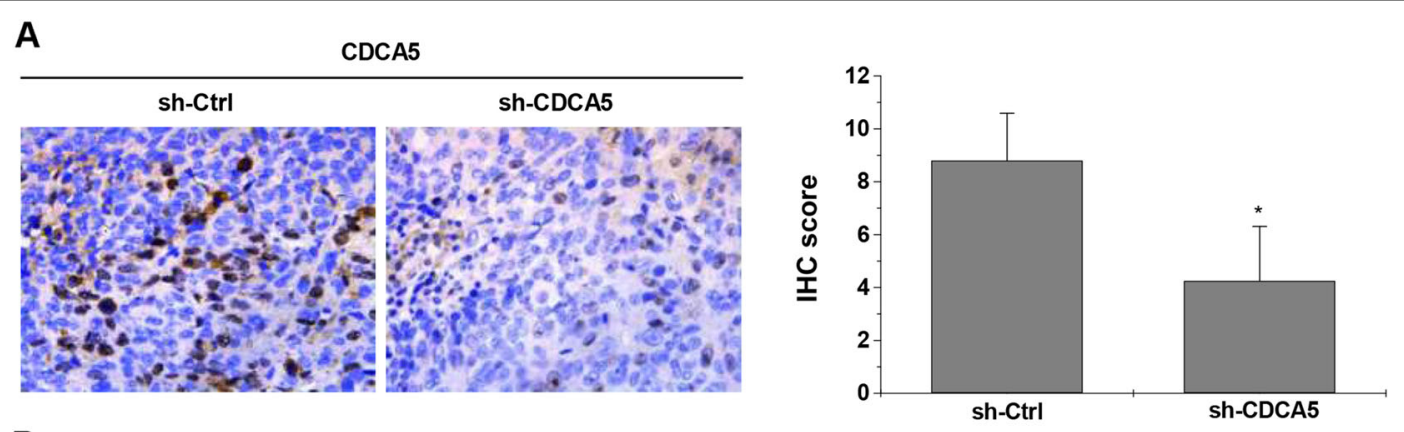

B
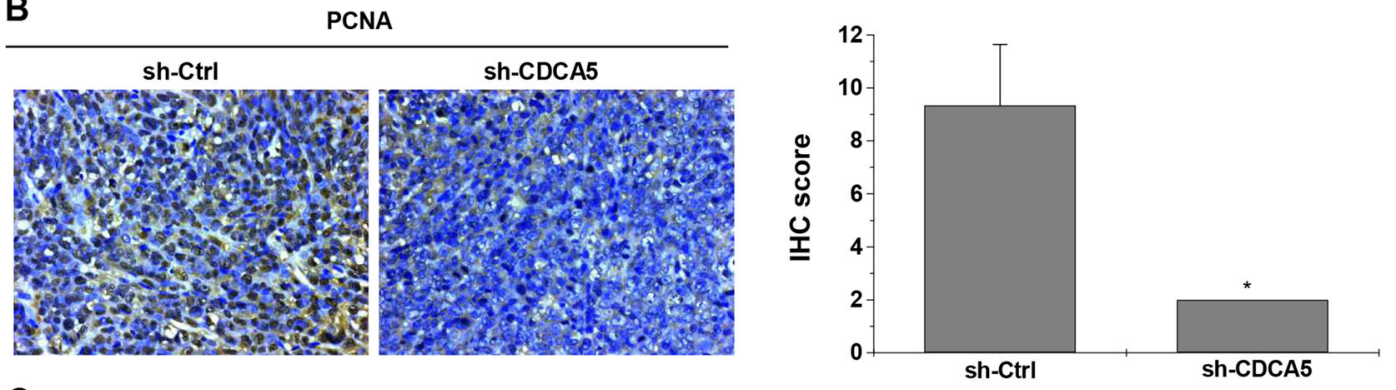

C

TUNEL
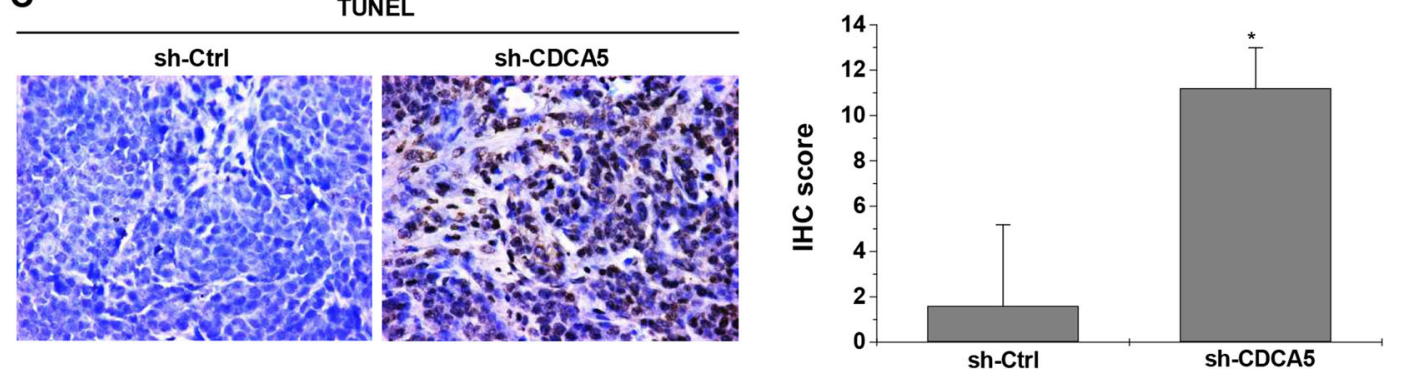

Fig. 7 CDCA5 knockdown inhibits cell proliferation and induces cell apoptosis in vivo. IHC was performed to detect CACA5 (a) and PCNA (b) expression in tumor sections, and TUNEL assay (c) was used to determine the apoptotic cells in tissues. The representative images of IHC or TUNELA assay (left panel, $\times 400)$ and statistical analysis of CDCA5 protein expression (right panel) are shown $\left({ }^{*} P<0.05 \mathrm{vs}\right.$. sh-Ctrl group). All experiments were performed in triplicate

\section{Cell lines and cell culture}

CRC cell lines (RKO, HCT-8, HT-29, HCT116, and Caco-2) were purchased from Cell Bank, Shanghai Institutes for Biological Sciences, Chinese Academy of Sciences (Shanghai, China). FHC (normal human colon cell line) was purchased from American Type Culture Collection (Manassas, VA, USA). RKO, HCT116, and HCT-8 were grown in RPMI-1640 (Gibco; Carlsbad, CA, USA), HT-29 in M5'A (KeyGEN; Jiangsu, China), Caco-2 in DMEM (Gibco), and FHC in DMEM:F12 (Gibco), at $37^{\circ} \mathrm{C}$ in a humidified atmosphere of $5 \% \mathrm{CO}_{2}$. All culture media contained $10 \%$ fetal bovine serum (FBS) (Gibco), 100 units $/ \mathrm{ml}$ penicillin and $100 \mathrm{mg} / \mathrm{ml}$ streptomycin (Hyclone; Logan, UT, USA).

\section{Lentivirus transduction}

Three independent shRNA targeting CDCA5 (shCDCA5-1, 2, 3-lentivirus-GFP; Cat\#: PIEL248072721) and a sh-Ctrl (sh-Ctrl-lentivirus-GFP, GV248; Cat\#:
LVCON077) were provided by Shanghai GeneChem Company (Shanghai, China). After cells were seeded in six-well plates, lentivirus encoding shRNA or control shRNA was added at the multiplicity of infection recommended by the manufacturer (MOI: 10). After 72-h incubation, CDCA5 expression was determined using qPCR and Western blot.

\section{Trypan blue exclusion and cell number counting}

Cells were stained using $0.4 \%$ trypan blue, and analyzed using a Countstar Automated Cell Counter (Inno-Alliance Biotech, Inc.; Wilmington, DE, USA).

\section{CCK-8 assay}

Cell viability was examined using the Cell Counting Kit-8 (Dojindo; Japan). Incubation lasted for $2 \mathrm{~h}$ at $37^{\circ} \mathrm{C}$. Absorbance was detected at $450 \mathrm{~nm}$ using Infinite 200 Pro microplate reader (Tecan; Männedorf, Switzerland). 

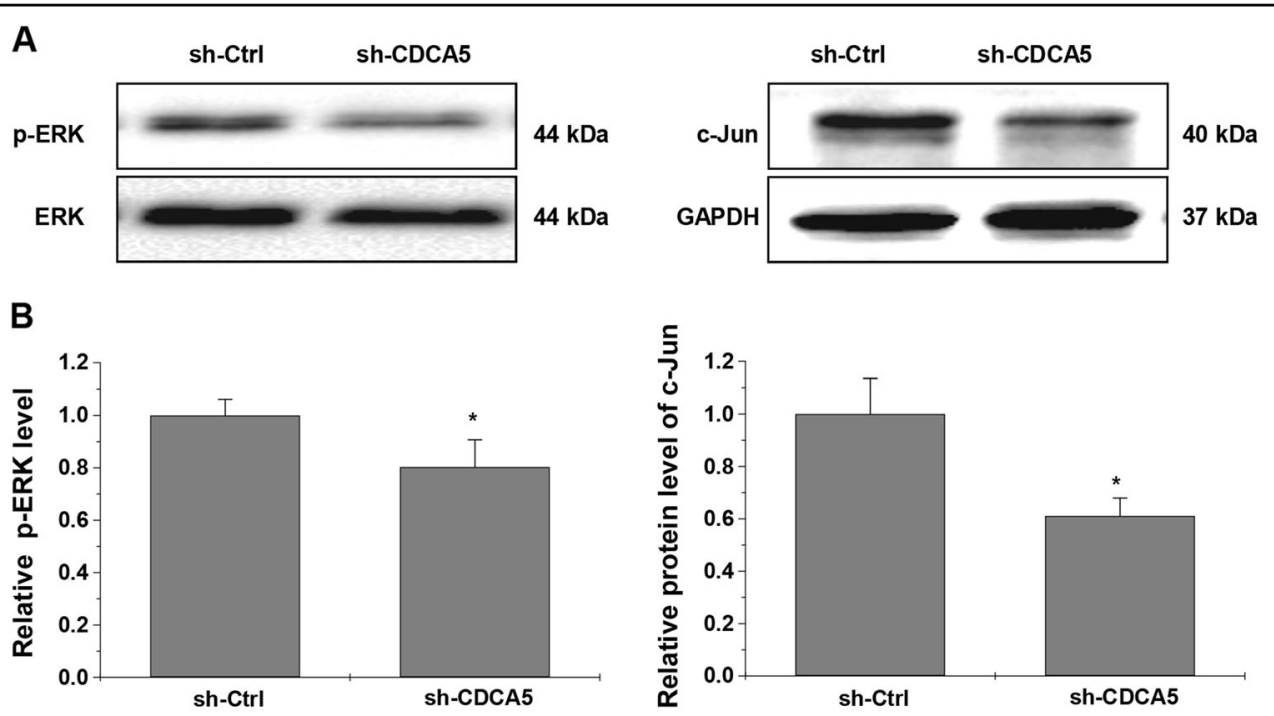

Fig. 8 CDCA5 knockdown suppresses activation of ERK signaling pathway. a Protein levels of p-ERK, ERK, and c-jun in HCT116 cells after transduction with shRNA-CDCA5 or sh-Ctrl lentiviurs, as determined by Western-blot analysis. $\mathbf{b}$ The integrated band density was determined using ImageLab Software, using GAPDH as the internal control. Gene expression is presented as the percentage relative to the sh-Ctrl group ( $\left.{ }^{*} P<0.05\right)$. All experiments were performed in triplicate

\section{Colony formation assay}

Cells were seeded into 12 -well plates (500 cells/well) and incubated in humidified air containing $5 \% \mathrm{CO}_{2}$ at $37^{\circ} \mathrm{C}$ for $10-12$ days. Culture medium was replaced every 2-3 days. The formed colonies were washed with phosphate-buffered saline, fixed with $4 \%$ formaldehyde and then stained with $0.01 \%$ crystal violet. The number of colonies was counted manually.

\section{Cell cycle analysis}

Cells were fixed with $70 \%$ ethanol at $4{ }^{\circ} \mathrm{C}$ for $12-16 \mathrm{~h}$, and stained with propidium iodide (PI; Thermo Fisher; Carlsbad, CA, USA). Percentage of cells in different cell cycle phases was analyzed using a FACS Caliber (BectonDickinson; San Jose, CA, USA).

\section{Hoechst33342 staining}

Cells were fixed with $4 \%$ formaldehyde and then stained with Hoechst dye (Beyotime, Jiangsu, China) for 15-20 min. Images were captured using a phase-contrast fluorescent microscope (Leica Microsystems; Wetzlar, Germany) at $\times 400$ magnification.

\section{Apoptosis analysis}

Cells were incubated with Annexin V-APC solution (KeyGEN) for $15 \mathrm{~min}$. The percentage of apoptotic was analyzed by flow cytometry (FACS Caliber, BectonDickinson).

\section{Caspase-3 activation analysis}

Caspase-3 activity was measured using a colorimetric assay (KeyGEN). Briefly, cell lysate (100- $\mu$ g protein) was mixed with $50-\mu \mathrm{l}$ specific caspase-3 substrate (Asp-GlueVal-Asp (DEAD)-pNA), and incubated for $2 \mathrm{~h}$. Absorbance was measured at $405 \mathrm{~nm}$. Caspase activity was presented relative to the control cells.

\section{In vivo experiments}

$\mathrm{BALB} / \mathrm{c}$ nude mice (male, 4-6 weeks of age,) were purchased from Shanghai SLAC Laboratory Animal Co. (Shanghai, China) and maintained in a specific pathogenfree facility. After transduction with sh-Ctrl or sh-CDCA5 lentivirus, CRC cells $\left(1 \times 10^{6}\right)$ suspended in 100- $\mu$ l FBSfree medium containing $50 \%$ matrigel were injected subcutaneously into the opposite flanks of mice $(n=5$; selfcontrol). Tumor growth was monitored every other day from day 5 after injection using a standard caliper. Tumor volume $\left(\mathrm{mm}^{3}\right)$ was calculated as: $1 / 2\left(\right.$ length $\times$ width $\left.^{2}\right)$, whereas length is the longest longitudinal diameter and width is the longest transverse diameter.

At the end of experiments, mice were anesthetized with isoflurane and fluorescent images of the tumors were obtained with an IVIS spectrum whole live-animal imaging system (PerkinElmer; Santa Clara, CA, USA). The mice were sacrificed and tumor tissues were collected, weighed, and processed for IHC staining. All animal experiments were approved by the Animal Committee of FJTCM. 


\section{Western-blot analysis}

Western-blot analysis was performed as described previously $^{36,37}$. Cell lysate was resolved by sodium dodecyl sulfate polyacrylamide gel electrophoresis $(10 \%)$ and then transferred onto nitrocellulose membranes. After blocking with $5 \%$ nonfat milk for $2 \mathrm{~h}$, the membranes were incubated with a primary antibody (dilution 1:1000) overnight at $4{ }^{\circ} \mathrm{C}$. All antibodies were from Cell Signaling Technology (CST; Beverly, MA, USA), except for CDCA5 and CDK1 (Abcam; Cambridge, MA, USA). After extensive washing, the membranes were incubated with a goat antirabbit HRP (horseradish peroxidase) secondary antibody (dilution 1:2000). Protein bands were detected with a chemiluminescence kit (Thermo Fisher), and analyzed using the ImageLab software. The expression of target proteins was normalized against GAPDH and presented as percentages of the control cells (sh-Ctrl or FHC cells).

\section{Immunohistochemistry}

Immunohistochemistry was performed as described previously ${ }^{38,39}$. Briefly, tissue sections were incubated with an antibody against CDCA5 (1:800 dilution; Abcam, ab192237) or PCNA (1:800 dilution; Abcam, ab18197). Background was determined by omitting the primary antibody. CDCA5 expression was determined using a scoring system described in detail in the Section "Tissues microarray and survival analysis".

\section{TUNEL assay}

Apoptotic cells in tissue sections were detected using TUNEL staining. The percentage of TUNEL-positive cells and staining intensity were evaluated using a scoring system described in detail in the Section "Tissues microarray and survival analysis".

\section{Statistics analysis}

All statistical analyses were conducted using SPSS 20.0 (SPSS Inc.). Continuous variables were analyzed using Student's $t$ test for independent or paired samples as appropriate for experiments involving two groups, and with one-way ANOVA for experiments involving three or more groups, and presented as mean \pm standard deviation. Survival data were analyzed using the Kaplan-Meier method and compared with log-rank test. $P<0.05$ (twosided) was considered statistically significant.

\section{Acknowledgments}

This study was supported by the National Natural Science Foundation of China (\#81673721 and 81803882), the International Cooperative Project of Fujian Department of Science and Technology (\#201710007) and the Chinese Government Scholarship from China Scholarship Council (\#[2016]3100). We thank Dr. Xiangfeng Wang from First People's Hospital Affiliated to Fujian University of Traditional Chinese Medicine and Dr. Yaodong Wang from Fujian Provincial Hospital for assistance in collection of human patient tissue samples. We thank Drs. Wei Lin and Weidong Zhu for helpful advice and discussions.

\begin{abstract}
Author details
${ }^{1}$ Academy of Integrative Medicine, Fujian University of Traditional Chinese Medicine, 1 Qiuyang Road, Minhou Shangjie, Fuzhou, Fujian 350122, China. ${ }^{2}$ Fujian Key Laboratory of Integrative Medicine on Geriatrics, Fujian University of Traditional Chinese Medicine, 1 Qiuyang Road, Minhou Shangjie, Fuzhou, Fujian 350122, China. ${ }^{3}$ Department of Pediatrics, Case Western Reserve University School of Medicine, Rainbow Babies and Children's Hospital, Cleveland, $\mathrm{OH}$ 44106, USA
\end{abstract}

\section{Conflict of interest}

The authors declare that they have no conflict of interest.

\section{Publisher's note}

Springer Nature remains neutral with regard to jurisdictional claims in published maps and institutional affiliations.

Supplementary Information accompanies this paper at (https://doi.org/ 10.1038/s41389-019-0123-5).

Received: 23 August 2018 Revised: 25 October 2018 Accepted: 22 January 2019

Published online: 26 February 2019

\section{References}

1. Siegel, R. L., Miller, K. D. \& Jemal, A. Cancer statistics, 2016. Cancer J. Clin. 66(1), 7-30 (2016).

2. Sherr, C. J. Cancer cell cycles. Science 274(5293), 1672-1677 (1996).

3. Hanahan, D. \& Weinberg, R. A. Hallmarks of cancer: the next generation. Cell 144(5), 646-674 (2011).

4. Hartwell, L. H. \& Kastan, M. B. Cell cycle control and cancer. Science 266(5192), 1821-1828 (1994).

5. Kastan, M. B. \& Bartek, J. Cell-cycle checkpoints and cancer. Nature 432(7015), 316-323 (2004)

6. Zhang, N. \& Pati, D. Sororin is a master regulator of sister chromatid cohesion and separation. Cell Cycle 11(11), 2073-2083 (2012).

7. Rankin, S., Ayad, N. G. \& Kirschner, M. W. Sororin a substrate of the anaphasepromoting complex is required for sister chromatid cohesionin vertebrates. Mol. Cell 18(2), 185-200 (2005).

8. Schmitz, J., Watrin, E., Lénárt, P., Mechtler, K. \& Peters, J. M. Sororin is required for stable binding of cohesin to chromatin and for sister chromatid cohesion in interphase. Curr. Biol. 17(7), 630-636 (2007).

9. Nishiyama, T. et al. Sororin mediates sister chromatid cohesion by antagonizing Wapl. Cell 143(5), 737-749 (2010).

10. Showe, M. K., Kossenkov, A. V. \& Showe, L. C. The peripheral immune response and lung cancer prognosis. Oncoimmunology 1(8), 1414-1416 (2012).

11. Nguyen, M. H. et al. Phosphorylation and activation of cell division cycle associated 5 by mitogen- activated protein kinaseplay a crucial role in human lung carcinogenesis. Cancer Res. 70(13), 5337-5347 (2010).

12. Bidkhori, G. et al. Reconstruction of an integrated genome-scale co-expression network reveals key modules involved in lung adenocarcinoma. PLOS ONE $\mathbf{8}$ (7), e67552 (2013)

13. Chang, I. W. et al. CDCA5 overexpression is an indicator of poor prognosis in patients with urothelial carcinomas of the upper urinary tract and urinary bladder. Am. J. Transl. Res. 7(4), 710-722 (2015).

14. Tokuzen, N., Nakashiro, K., Tanaka, H., Iwamoto, K. \& Hamakawa, H. Therapeutic potential of targeting cell division cycle associated 5 for oral squamous cell carcinoma. Oncotarget 7(3), 2343-2353 (2015).

15. Qi, F. et al. Pien Tze Huang inhibits the proliferation, and induces the apoptosis and differentiation of colorectal cancer stem cells via suppression of the Notch1 pathway. Oncol. Rep. 35(1), 511-517 (2016).

16. Cheng, W. et al. Downregulation of HP1a suppresses proliferation of cholangiocarcinoma by restoring SFRP1 expression. Oncotarget 7(30), 48107-48119 (2016).

17. Liang, Y. et al. Aryl hydrocarbon receptor nuclear translocator is associated with tumor growth and progression of hepatocellular carcinoma. Int. J. Cancer 130(8), 1745-1754 (2012). 
18. Skrzypczak, M. et al. Modeling oncogenic signaling in colon tumors by multidirectional analyses of microarray data directed for maximization of analytical reliability. PLOS ONE 5(10), e13091 (2010).

19. Matsuyama, T. et al. MUC12 mRNA expression is an independent marker of prognosis in stage II and stage III colorectal cancer. Int. J. Cancer 127(10), 2292-2299 (2010)

20. Uddin, S. et al. Genome-wide expression analysis of Middle Eastern colorectal cancer reveals FOXM1 as a novel target for cancer therapy. Am. J. Pathol. 178 (2), 537-547 (2011).

21. Li, C., Li, X., Wu, L. \& Jiang, Z. Elevated AQP1 expression is associated with unfavorable oncologic outcome in patients with hilar cholangiocarcinoma. Technol. Cancer Res. Treat. 16(4), 421-427 (2017).

22. Shen, A. et al. Pien Tze Huang overcomes multidrug resistance and epithelial-mesenchymal transition in human colorectal carcinoma cells via suppression of TGF- $\beta$ pathway. Evid. Based Complement. Altern. Med. 2014 679436 (2014)

23. Chen, $\mathrm{H}$. et al. Pien Tze Huang inhibits hypoxia-induced epithelial mesenchymal transition in human colon carcinoma cells through suppression of the HIF-1 pathway. Exp. Ther. Med. 7(5), 1237-1242 (2014).

24. Shen, A. et al. Pien Tze Huang inhibits tumor angiogenesis in a mouse model of colorectal cancer via suppression of multiple signaling pathways. Oncol. Rep. 30(3), 1701-1706 (2013).

25. Chen, L. et al. Patrinia scabiosaefolia inhibits colorectal cancer growth through suppression of tumor angiogenesis. Oncol. Rep. 30(3), 1439-1443 (2013).

26. Raman, M., Chen, W. \& Cobb, M. H. Differential regulation and properties of MAPKs. Oncogene 26(22), 3100-3112 (2007)

27. Cargnello, M. \& Roux, P. P. Activation and function of the MAPKs and their substrates, the MAPK-activated protein kinases. Microbiol. Mol. Biol. Rev. 75 (2), 50-83 (2011).

28. Weng, Y. R. et al. The role of ERK2 in colorectal carcinogenesis is partly regulated by TRAPPC4. Mol. Carcinog. 53(Suppl 1), E72-E84 (2014).
29. Tai, C. J. et al. Clinical-pathological correlation of K-Ras mutation and ERK phosphorylation in colorectal cancer. Pol. J. Pathol. 63(2), 93-100 (2012).

30. Wang, Z. et al. Cyclin B1/Cdk1 coordinates mitochondrial respiration for cellcycle G2/M progression. Dev. Cell. 29(2), 217-232 (2014).

31. Jang, S. H., Kim, A. R., Park, N. H., Park, J. W. \& Han, I. S. DRG2 regulates G2/ M progression via the cyclin B1-Cdk1 complex. Mol. Cells 39(9), 699-704 (2016).

32. Peng, J. et al. Oligomerization of membrane-bound $\mathrm{BCl}-2$ is involved in its pore formation induced by tBid. Apoptosis 14(10), 1145-1153 (2009).

33. Peng, J. et al. tBID elicits a conformational alteration in membrane-bound BCl2 such that it inhibits Bax Pore formation. J. Biol. Chem. 281, 35802-35811 (2006).

34. Zhang, W. \& Liu, H. T. MAPK signal pathways in the regulation of cell proliferation in mammalian cells. Cell Res. 12(1), 9-18 (2002).

35. Yang, $Y$. et al. Functional roles of p38 mitogen-activated protein kinase in macrophage-mediated inflammatory responses. Mediat. Inflamm. 2014 352371 (2014).

36. Izrailit, J., Berman, H. K., Datti, A., Wrana, J. L. \& Reedijk, M. High throughput kinase inhibitor screensreveal TRB3 and MAPK-ERKTGF $\beta$ pathways as fundamental Notch regulators in breast cancer. Proc. Natl Acad. Sci. U SA 110(5), 1714-1719 (2013).

37. Charles, N. J., Thomas, P. \& Lange, C. A. Expression of membrane progesterone receptors (mPR/PAQR) in ovarian cancer cells: implications for progesteroneinduced signaling events. Horm. Cancer 1(4), 167-176 (2010).

38. Setia, S., Nehru, B. \& Sanyal, S. N. Upregulation of MAPK/Erk and PI3K/Akt pathways in ulcerative colitis-associated colon cancer. Biomed. Pharmacother. 68(8), 1023-1029 (2014).

39. Peng, $Y$. et al. Angiogenin interacts with ribonuclease inbibitor regulating PI3K/Akt/mTOR signaling pathway in bladder cancer cells. Cell Signal. 26(12), 2782-2792 (2014) 